\title{
Double Barrier Hitting Time Distribution of a Mean-reverting Lognormal Process and Its Application to Pricing Exotic Options*
}

\author{
C.F. Lo ${ }^{\dagger \ddagger}$, T.K. Chung ${ }^{\S}$ and C.H. Huiף
}

\begin{abstract}
In this paper we propose a simple and easy-to-use method for computing accurate estimate (in closed form) of the double barrier hitting time distribution of a mean-reverting lognormal process, and discuss its application to pricing exotic options whose payoffs are contingent upon barrier hitting times. This new approach is also able to provide tight upper and lower bounds (in closed form) of the exact result. Within the multi-stage approximation scheme, the estimate and bounds can be easily improved in a systematic manner. Furthermore, this approach can be straightforwardly extended to those cases with specified moving boundaries as well.
\end{abstract}

Keywords: First hitting time; mean-reverting lognormal process; barrier options; method of images.

\section{Introduction}

In the past decade barrier options have become very popular instruments for a wide variety of hedging and investment in foreign exchange, equity and commodity markets, largely in the over-the-counter markets. An advantage of trading barrier options is that they provide more flexibility in tailoring the portfolio

\footnotetext{
${ }^{*}$ The conclusions herein do not represent the views of the Hong Kong Monetary Authority.

†Author for correspondence. Institute of Theoretical Physics and Department of Physics, The Chinese University of Hong Kong, Shatin, New Territories, Hong Kong, China (Email: cflo@phy.cuhk.edu.hk)

${ }^{\ddagger}$ Hong Kong Institute for Monetary Research, Hong Kong Monetary Authority, 55th Floor, Two International Finance Centre, 8 Finance Street, Hong Kong, China

$\S$ Department of Physics, The Chinese University of Hong Kong, Shatin, New Territories, Hong Kong, China (Email: tkchung@phy.cuhk.edu.hk)

\Research Department, Hong Kong Monetary Authority, 55th Floor, Two International Finance Centre, 8 Finance Street, Hong Kong, China (Email: chhui@hkma.gov.hk)
}

returns while lowering the cost of option premiums. In the commodity and foreign exchange markets empirical studies show that the mean-reverting lognormal process (MRL-process) provides a more accurate description of the commodity prices and exchange rates than the lognormal process. However, unlike the standard barrier options for a lognormal process, the valuation of barrier options for a MRL-process poses a real challenge for there is no exact analytical solution available (Lo and Hui 2006).

Recently, based upon the method of images, Hui and Lo (2006) were able to develop an efficient analytical approach to provide accurate estimates of single-barrier options for a MRL-process. In this paper we generalize their method to compute the double barrier hitting time distribution function for a MRL-process. Based upon the exact double barrier hitting time distribution function (in closed form) of the MRL-process with two parametric moving barriers, we are able to obtain very accurate estimate (in closed form) of the desired distribution function associated with two fixed barriers, including the upper and lower bounds (in closed form) of the exact result. With the multi-stage approximation scheme, the estimate and bounds can also be systematically improved in a straightforward manner. These results are applicable to the analysis of the MRL-process for various mean-reverting financial variables (Hui and Lo 2006; Sorensen 1997); for instance, we can apply the results to value some exotic options whose payoffs are contingent upon barrier hitting times.

\section{First Hitting Time Distribution}

We consider a MRL-process described by the stochastic differential equation

$$
\begin{aligned}
d S= & \left\{\mu(t)+\kappa(t)\left(\ln S_{m}(t)-\ln S\right)\right\} S d t+ \\
& \sigma(t) S d W_{t}
\end{aligned}
$$


where $S$ is the underlying, $\kappa(t)$ is the mean-reverting force, $S_{m}(t)$ is the equilibrium position, $\mu(t)$ is the drift term, $\sigma(t)$ is the volatility and $W_{t}$ is a standard Weiner process. Under the standard transfomation $x=\ln \left(S / S_{L}\right)$ and $x_{m}=\ln \left(S_{m} / S_{L}\right)$, we could re-write the FPE as

$$
\begin{aligned}
d x= & \left\{\mu(t)+\kappa(t)\left[x_{m}(t)-x\right]-\frac{1}{2} \sigma(t)^{2}\right\} d t+ \\
& \sigma(t) d W_{t},
\end{aligned}
$$

and the associated Fokker-Planck equation (FPE) governing the transition probability density function (p.d.f.) is given by

$$
\begin{aligned}
& \frac{\partial P(x, t)}{\partial t} \\
= & \frac{1}{2} \sigma(t)^{2} \frac{\partial^{2} P(x, t)}{\partial x^{2}}- \\
& \left\{\mu(t)+\kappa(t)\left[x_{m}(t)-x\right]-\frac{1}{2} \sigma(t)^{2}\right\} \times \\
& \frac{\partial P(x, t)}{\partial x}+\kappa(t) P(x, t) .
\end{aligned}
$$

It is straightforward to show that its solution corresponding to the so-called natural boundary condition is given by

$$
P(x, t)=\int_{-\infty}^{\infty} K\left(x, t ; x^{\prime}, 0\right) P\left(x^{\prime}, 0\right) d x^{\prime}
$$

where

$$
\begin{aligned}
& K\left(x, t ; x^{\prime}, 0\right) \\
= & \frac{1}{\sqrt{4 \pi \eta(t)}} \exp \left\{-\frac{\left[x e^{\lambda(t)}+\gamma(t)-x^{\prime}\right]^{2}}{4 \eta(t)}+\lambda(t)\right\} \\
\eta(t)= & \int_{0}^{t} \frac{1}{2} \sigma^{2}(s) e^{2 \lambda(s)} d s \\
\gamma(t)= & \int_{0}^{t}\left(\frac{1}{2} \sigma(s)^{2}-\mu(s)-\kappa(s) x_{m}(s)\right) e^{\lambda(s)} d s \\
\lambda(t)= & \int_{0}^{t} \kappa(s) d s .
\end{aligned}
$$

Given the initial condition $P(x, 0)=\delta\left(x-x_{0}\right)$, $P(x, t)=K\left(x, t ; x_{0}, 0\right)$ gives the unrestricted p.d.f. from $x_{0}$ to $x$.

In the presence of two absorbing barriers at $S=S_{L}$ and $S=S_{U}, S_{L} \leq S_{U}$, one has to impose the boundary conditions: $P(0, t)=P\left(L_{0}, t\right)=0$, with $0 \leq x_{0} \leq \ln \left(S_{U} / S_{L}\right) \equiv L_{0}$. By the method of images we are able to derive analytically the restricted p.d.f. (in closed form) with two moving absorbing boundaries as follows:

$$
\begin{aligned}
P^{D B}\left(x, t ; x_{0}, 0\right) & =\int_{0}^{L_{0}} G\left(x, t ; x^{\prime}, 0\right) P\left(x^{\prime}, 0\right) d x^{\prime} \\
& =G\left(x, t ; x_{0}, 0\right)
\end{aligned}
$$

where

$$
\begin{aligned}
G\left(x, t ; x^{\prime}, 0\right) & \sum_{n=-\infty}^{\infty}\left\{K\left(x, t ; x^{\prime}-2 n L_{0}, 0\right) e^{n(\beta-\alpha) x^{\prime}}-\right. \\
& \left.K\left(x, t ;-x^{\prime}-2 n L_{0}, 0\right) e^{-\{(n+1) \beta-n \alpha\} x^{\prime}}\right\} \times \\
& e^{-n \beta L_{0}-n^{2}(\beta-\alpha)} .
\end{aligned}
$$

The trajectories of the two moving boundaries are specified by

$$
\begin{aligned}
x_{L}^{*}(t) & \equiv \ln \left(\frac{S_{L}^{*}(t)}{S_{L}}\right)=[-\gamma(t)-\beta \eta(t)] e^{-\lambda(t)} \\
x_{U}^{*}(t) & \equiv \ln \left(\frac{S_{U}^{*}(t)}{S_{L}}\right) \\
& =\left[-\gamma(t)-\alpha \eta(t)+L_{0}\right] e^{-\lambda(t)}
\end{aligned}
$$

at any time $t \geq 0$, where $\beta$ and $\alpha$ are two real adjustable parameters controlling the movement of the two barriers. The corresponding first hitting time distribution function or first passage time distribution function (FPTDF) could be formulated as:

$$
\begin{aligned}
P_{\text {exit }}\left(x_{0}, t\right)= & 1-\int_{x_{L}^{*}(t)}^{x_{U}^{*}(t)} G\left(x, t ; x_{0}, 0\right) d x \\
= & \sum_{n=-\infty}^{\infty} e^{-n \beta L_{0}-n^{2}(\beta-\alpha) L_{0}} \times \\
& \left\{N\left[\frac{-\alpha \eta(t)-x_{0}+(2 n+1) L_{0}}{\sqrt{2 \eta(t)}}\right] \times\right. \\
& e^{n(\beta-\alpha) x_{0}}- \\
& N\left[\frac{-\alpha \eta(t)+x_{0}+(2 n+1) L_{0}}{\sqrt{2 \eta(t)}}\right] \times \\
& e^{\left.-\{(n+1) \beta-n \alpha\} x_{0}\right\}-} \\
& N\left[\frac{-\beta \eta(t)-x_{0}+2 n L_{0}}{\sqrt{2 \eta(t)}}\right] \times \\
& e^{n(\beta-\alpha) x_{0}}+. \\
& N\left[\frac{-\beta \eta(t)+x_{0}+2 n L_{0}}{\sqrt{2 \eta(t)}}\right] \times \\
& e^{\left.-\{(n+1) \beta-n \alpha\} x_{0}\right\}} .
\end{aligned}
$$


Here $N(\cdot)$ is the cumulative normal distribution function.

To simulate fixed upper and lower barriers, one could choose the optimal values of the adjustable parameters $\alpha$ and $\beta$ in such a way that both of the integrals

$$
\int_{0}^{t}\left[x_{U}^{*}(s)-L_{0}\right]^{2} d s \text { and } \int_{0}^{t}\left[x_{L}^{*}(s)\right]^{2} d s
$$

are minimum. In other words, we try to minimize the deviations from the fixed barriers by varying the parameters $\alpha$ and $\beta$. Simple algebraic manipulations then yield the optimal values of $\beta$ and $\alpha$ as follows:

$$
\begin{aligned}
\alpha_{\mathrm{opt}} & =-\frac{\int_{0}^{t}\left\{\gamma(s)+\left(e^{\lambda(s)}-1\right) L_{0}\right\} \eta(s) e^{-2 \lambda(s)} d s}{\int_{0}^{t} \eta^{2}(s) e^{-2 \lambda(s)} d s} \\
\beta_{\mathrm{opt}} & =-\frac{\int_{0}^{t} \gamma(s) \eta(s) e^{-2 \lambda(s)} d s}{\int_{0}^{t} \eta^{2}(s) e^{-2 \lambda(s)} d s}
\end{aligned}
$$

It should be noted that the above scheme could also be easily applied to those cases with time-dependent barriers, e.g. two exponentially moving boundaries, by choosing appropiate values of $\alpha$ and $\beta$. Furthermore, within the framework of this new approach, we can determine the upper and lower bounds for the exact barrier option prices too. It is not difficult to show ${ }^{1}$ that if the moving barriers stay outside the region bounded by the fixed barriers, i.e. $S_{U}^{*}(t)>S_{U}$ and $S_{L}^{*}(t)<S_{L}$, for the duration of interest, then the restricted p.d.f. (and the corresponding option price) will provide an upper bound for the exact value. On the other hand, if the moving barriers are embedded inside the bounded region, i.e. $S_{U}^{*}(t)<S_{U}$ and $S_{L}^{*}(t)>S_{L}$, then the p.d.f. (and the corresponding option price) will serve as a lower bound.

For illustration, we apply the approximation to evaluate the FPTDF associated with two fixed barriers located at $S_{U}=110$ and $S_{L}=90$ after a duration $T=0.25$. The current value of the underlying is $S=100$, and other input parameters are: $\kappa=0.5$, $\mu=0, \sigma=0.1$ and $S_{m}=100$. First of all, we determine the optimal values of the adjustable parameters $\alpha$ and $\beta$ :

$$
\begin{aligned}
& \alpha_{\mathrm{opt}}=-10.03765 \\
& \beta_{\mathrm{opt}}=9.089295 .
\end{aligned}
$$

\footnotetext{
${ }^{1}$ The proof is based upon the maximum principle for parabolic differential equations (see the appendix of Lo et al. (2003) for the relevant proof).
}

An estimate of the exact FPTDF can be evalutaed using Eq.(11) :

$$
P_{\text {exit }}(S=100, T=0.25)=0.073662
$$

As a check, the Crank-Nicolson method is used to numerically solve the FPE, and the (numerically) exact value of the FPTDF is given by

$$
P_{\text {exit }}^{\text {exact }}(S=100, T=0.25)=0.073955
$$

The approximate estimate is indeed very close to the exact result with an error of $0.40 \%$ only. Moreover, the corresponding upper and lower bounds are also evaluated as follows:

$$
\begin{aligned}
& \text { Upper Bound }=0.074298 \\
& \text { Lower Bound }=0.071471
\end{aligned}
$$

Clearly, the new approach is able to give very tight upper and lower bounds for the exact FPTDF with percentage error less than $3.5 \%$.

In order to systematically tighten the upper and lower bounds, we can adopt the multi-stage approximation scheme proposed by Lo et al. (2003). The essence of the approximation scheme is to replace each of the above smooth moving barriers by a continuous and piecewise smooth trajectory in order that the deviation from the fixed barrier is minimized in a systematic manner. As demonstrated by Lo et al. (2003), we then need to perform some simple onedimensional numerical integrations (e.g. using the Gauss quadrature method $)^{2}$ at the connecting points of each piecewise smooth barrier in order to evaluate the upper and lower bounds of the option price. As expected, the multi-stage approximation for both the upper and lower bounds becomes better and better as the number $N$ of stages increases; in fact, the gap between the bounds is asymptotically reduced to zero. In practice even a rather low-order approximation can yield very tight upper and lower bounds to the exact results, as demonstrated in Table 1 and Table 2.

\section{Application in Pricing Exotic Options}

The aforementioned FPTDF has a wide application in pricing exotic options whose payoffs are contingent upon barrier hitting times. For demonstration, we explicitly derive the price formulae for the double digital option and the double knockout call option as follows:

\footnotetext{
${ }^{2}$ The integration can be performed analytically and the result can be expressed in closed form in terms of the cumulative multi-variate normal distribution functions. However, in practice the numerical integrations are indeed very efficient.
} 


\section{Double-barrier Digital Option}

A European double-digital option pays one dollar if the underlying asset price stays within the two prescribed barriers until the option maturity $T$. Thus the price function is simply the survival probability of the underlying inside the two barriers, with an appropriate discount factor:

$$
\begin{aligned}
& P_{\text {double digital }}(S, T) \\
& =e^{-r T}\left[1-P_{\text {exit }}\left(\ln \left(\frac{S}{S_{L}}\right), T\right)\right] \\
& =e^{-r T} \sum_{n=-\infty}^{\infty}\left(\frac{S_{U}}{S_{L}}\right)^{-n^{2}(\beta-\alpha)-n \beta} \times \\
& \left\{N\left(\theta_{1}\right)\left(\frac{S}{S_{L}}\right)^{n(\beta-\alpha)}-\right. \\
& N\left(\theta_{2}\right)\left(\frac{S}{S_{L}}\right)^{-\{(n+1) \beta-n \alpha\}}- \\
& N\left(\theta_{3}\right)\left(\frac{S}{S_{L}}\right)^{n(\beta-\alpha)}+ \\
& \left.N\left(\theta_{4}\right)\left(\frac{S}{S_{L}}\right)^{-\{(n+1) \beta-n \alpha\}}\right\}
\end{aligned}
$$

where

$$
\begin{aligned}
\theta_{1}= & \frac{1}{\sqrt{2 \eta(T)}}\left\{-\alpha \eta(T)-\ln \left(\frac{S}{S_{L}}\right)+\right. \\
& \left.(2 n+1) \ln \left(\frac{S_{U}}{S_{L}}\right)\right\} \\
\theta_{2}= & \frac{1}{\sqrt{2 \eta(T)}}\left\{-\alpha \eta(T)+\ln \left(\frac{S}{S_{L}}\right)+\right. \\
& \left.(2 n+1) \ln \left(\frac{S_{U}}{S_{L}}\right)\right\} \\
\theta_{3}= & \frac{1}{\sqrt{2 \eta(T)}}\left\{-\beta \eta(T)-\ln \left(\frac{S}{S_{L}}\right)+\right. \\
& \left.2 n \ln \left(\frac{S_{U}}{S_{L}}\right)\right\} \\
\theta_{4}= & \frac{1}{\sqrt{2 \eta(T)}}\left\{-\beta \eta(T)+\ln \left(\frac{S}{S_{L}}\right)+\right. \\
& \left.2 n \ln \left(\frac{S_{U}}{S_{L}}\right)\right\} .
\end{aligned}
$$

\section{Double-barrier Knockout Call Option}

The option price is formulated as the discounted expected payoff with respect to the restricted p.d.f.:

$$
\begin{aligned}
& P_{\text {double knockout call }}(S, T) \\
& =e^{-r T} E\left[\max \left(S_{L} e^{x}-K, 0\right)\right] \\
& =e^{-r T} \int_{x_{L}^{*}(T)}^{x_{U}^{*}(T)} G\left(x, T ; x_{0}, 0\right) \times \\
& \max \left(S_{L} e^{x}-K, 0\right) d x \\
& =e^{-r T}\left\{S_{L} \int_{\ln \left(\frac{K}{S_{L}}\right)}^{x_{U}^{*}(T)} G\left(x, T ; x_{0}, 0\right) e^{x} d x-\right. \\
& \left.K \int_{\ln \left(\frac{K}{S_{L}}\right)}^{x_{U}^{*}(T)} G\left(x, T ; x_{0}, 0\right) d x\right\} \\
& =e^{-r T} \sum_{n=-\infty}^{\infty}\left(\frac{S_{U}}{S_{L}}\right)^{-n^{2}(\beta-\alpha)-n \beta} \times \\
& \left\{\exp \left(\left[\ln \left(\frac{S}{S_{L}}\right)-2 n \ln \left(\frac{S_{U}}{S_{L}}\right)\right] e^{-\lambda T}\right)\right. \\
& \times S_{L} \exp \left\{\eta(T) e^{-2 \lambda T}-\gamma(T) e^{-\lambda T}\right\} \\
& \times\left[N\left(\theta_{1}\right)\left(\frac{S}{S_{L}}\right)^{n(\beta-\alpha)}-\right. \\
& \left.N\left(\theta_{3}\right)\left(\frac{S}{S_{L}}\right)^{n(\beta-\alpha)}\right]- \\
& \exp \left(\left[\ln \left(\frac{S_{L}}{S}\right)-2 n \ln \left(\frac{S_{U}}{S_{L}}\right)\right] e^{-\lambda T}\right) \\
& \times S_{L} \exp \left\{\eta(T) e^{-2 \lambda T}-\gamma(T) e^{-\lambda T}\right\} \\
& \times\left[N\left(\theta_{2}\right)\left(\frac{S}{S_{L}}\right)^{-\{(n+1) \beta-n \alpha\}}-\right. \\
& \left.N\left(\theta_{4}\right)\left(\frac{S}{S_{L}}\right)^{-\{(n+1) \beta-n \alpha\}}\right]- \\
& K\left[N\left(\theta_{5}\right)\left(\frac{S}{S_{L}}\right)^{n(\beta-\alpha)}-\right. \\
& \left.N\left(\theta_{7}\right)\left(\frac{S}{S_{L}}\right)^{n(\beta-\alpha)}\right]+ \\
& K\left[N\left(\theta_{6}\right)\left(\frac{S}{S_{L}}\right)^{-\{(n+1) \beta-n \alpha\}}-\right. \\
& \left.\left.N\left(\theta_{8}\right)\left(\frac{S}{S_{L}}\right)^{-\{(n+1) \beta-n \alpha\}}\right]\right\}
\end{aligned}
$$

where

$$
\begin{aligned}
\theta_{1}= & \frac{1}{\sqrt{2 \eta(T)}}\left\{-\alpha \eta(T)-\ln \left(\frac{S}{S_{L}}\right)+\right. \\
& \left.(2 n+1) \ln \left(\frac{S_{U}}{S_{L}}\right)-2 \eta(T) e^{-\lambda T}\right\}
\end{aligned}
$$




$$
\begin{aligned}
& \theta_{2}=\frac{1}{\sqrt{2 \eta(T)}}\left\{-\alpha \eta(T)+\ln \left(\frac{S}{S_{L}}\right)+\right. \\
& \left.(2 n+1) \ln \left(\frac{S_{U}}{S_{L}}\right)-2 \eta(T) e^{-\lambda T}\right\} \\
& \theta_{3}=\frac{1}{\sqrt{2 \eta(T)}}\left\{\ln \left(\frac{K}{S_{L}}\right) e^{\lambda T}-\ln \left(\frac{S}{S_{L}}\right)+\right. \\
& \left.\gamma(T)+2 n \ln \left(\frac{S_{U}}{S_{L}}\right)-2 \eta(T) e^{-\lambda T}\right\} \\
& \theta_{4}=\frac{1}{\sqrt{2 \eta(T)}}\left\{\ln \left(\frac{K}{S_{L}}\right) e^{\lambda T}+\ln \left(\frac{S}{S_{L}}\right)+\right. \\
& \left.\gamma(T)+2 n \ln \left(\frac{S_{U}}{S_{L}}\right)-2 \eta(T) e^{-\lambda T}\right\} \\
& \theta_{5}=\frac{1}{\sqrt{2 \eta(T)}}\left\{-\alpha \eta(T)-\ln \left(\frac{S}{S_{L}}\right)+\right. \\
& \left.(2 n+1) \ln \left(\frac{S_{U}}{S_{L}}\right)\right\} \\
& \theta_{6}=\frac{1}{\sqrt{2 \eta(T)}}\left\{-\alpha \eta(T)+\ln \left(\frac{S}{S_{L}}\right)+\right. \\
& \left.(2 n+1) \ln \left(\frac{S_{U}}{S_{L}}\right)\right\} \\
& \theta_{7}=\frac{1}{\sqrt{2 \eta(T)}}\left\{\ln \left(\frac{K}{S_{L}}\right) e^{\lambda T}-\ln \left(\frac{S}{S_{L}}\right)+\right. \\
& \left.\gamma(T)+2 n \ln \left(\frac{S_{U}}{S_{L}}\right)\right\} \\
& \theta_{8}=\frac{1}{\sqrt{2 \eta(T)}}\left\{\ln \left(\frac{K}{S_{L}}\right) e^{\lambda T}+\ln \left(\frac{S}{S_{L}}\right)+\right. \\
& \left.\gamma(T)+2 n \ln \left(\frac{S_{U}}{S_{L}}\right)\right\} .
\end{aligned}
$$

\section{References}

1. Hui, C.H. and C.F. Lo (2006): "Currency barrier option pricing with mean reversion", Journal of Futures Markets, 26(10): 939-958.

2. Lo, C.F., H.C. Lee and C.H. Hui (2003): "A simple approach for pricing barrier options with time-dependent parameters", Quantitative Finance 3: 98-107.

3. Lo, C.F. and C.H. Hui (2006): "Computing the first passage time density of a timedependent Ornstein-Uhlenbeck process to a moving boundary", Applied Mathematics Letters 19:1399-1405.

4. Sorensen, C. (1997): "An equilibrium approach to pricing foreign currency options", European Financial Management 3:63-85.

\section{Conclusion}

In this paper we have presented a simple and easyto-use method to compute the double barrier hitting time distribution function for a mean-reverting lognormal process and discussed its application to pricing exotic options whose payoffs are contingent on barrier hitting times. This new approach is able to yield very accurate estimate of the desired distribution function, including the upper and lower bounds of the exact result. With the multi-stage approximation scheme, the estimate and bounds can be systematically improved in a straightforward manner as well. Moreover, it is natural that by tuning the parameters $\alpha$ and $\beta$ the approach can be applied to determine the distribution functions of those cases with specifed moving barriers. 
Proceedings of the World Congress on Engineering 2007 Vol II

WCE 2007, July 2 - 4, 2007, London, U.K.

Table 1. Comparison of estimates and bounds of the first passage time distribution function (FPTDF) with the (numerically) exact results by $\mathrm{CN}$ method. Percentage error is defined as (estimate or bound $-\mathrm{CN}$ result)/ $\mathrm{CN}$ result $\times 100 \%$. Number of images summed: $\mathrm{n}=-2$ to $\mathrm{n}=2$. Input parameters are: $\mathrm{k}=0.5, \mu=0, \sigma=0.1, \mathrm{~S}_{\mathrm{u}}=110, \mathrm{~S}_{\mathrm{L}}=90, \mathrm{~S}_{\mathrm{m}}=100$ and $\mathrm{S}=100$.

\begin{tabular}{|c|c|c|c|c|c|c|}
\hline \multirow{2}{*}{ Duration } & \multirow{2}{*}{$\begin{array}{l}\text { Crank-Nicolson } \\
\begin{array}{c}\Delta \mathrm{t}=0.0001 \\
\Delta \mathrm{x}=0.0001\end{array}\end{array}$} & \multicolumn{3}{|c|}{$\begin{array}{c}\text { Single-Stage Approximation } \\
\text { (\%error) }\end{array}$} & \multicolumn{2}{|c|}{$\begin{array}{c}\text { Multistage Approximation } \\
\text { (\%error) }\end{array}$} \\
\hline & & Optimal Track & Upper Bound & Lower Bound & Two Stage & Four Stage \\
\hline $\mathrm{T}=0.25$ & 0.073955 & $\begin{array}{l}0.073662 \\
(-0.40 \%)\end{array}$ & $\begin{array}{c}0.074298 \\
(0.46 \%)\end{array}$ & $\begin{array}{l}0.071471 \\
(-3.36 \%)\end{array}$ & $\begin{array}{c}0.074054 \\
(0.13 \%)\end{array}$ & $\begin{array}{c}0.073982 \\
(0.04 \%)\end{array}$ \\
\hline $\mathrm{T}=0.5$ & 0.261564 & $\begin{array}{l}0.260472 \\
(-0.42 \%)\end{array}$ & $\begin{array}{c}0.265238 \\
(1.40 \%)\end{array}$ & $\begin{array}{l}0.245338 \\
(-6.20 \%)\end{array}$ & $\begin{array}{c}0.262481 \\
(0.35 \%)\end{array}$ & $\begin{array}{c}0.261805 \\
(0.09 \%)\end{array}$ \\
\hline $\mathrm{T}=0.75$ & 0.422641 & $\begin{array}{l}0.421608 \\
(-0.24 \%)\end{array}$ & $\begin{array}{c}0.433553 \\
(2.58 \%)\end{array}$ & $\begin{array}{l}0.384377 \\
(-9.05 \%)\end{array}$ & $\begin{array}{c}0.425285 \\
(0.63 \%)\end{array}$ & $\begin{array}{c}0.423315 \\
(0.16 \%)\end{array}$ \\
\hline $\mathrm{T}=1$ & 0.549373 & $\begin{array}{l}0.549643 \\
(0.05 \%)\end{array}$ & $\begin{array}{l}0.570380 \\
(3.82 \%)\end{array}$ & $\begin{array}{l}0.487778 \\
(-11.21 \%)\end{array}$ & $\begin{array}{l}0.554486 \\
(0.93 \%)\end{array}$ & $\begin{array}{l}0.550653 \\
(0.23 \%)\end{array}$ \\
\hline
\end{tabular}

Table 2. Comparison of estimates and bounds of the first passage time distribution function (FPTDF) with the (numerically) exact results by $\mathrm{CN}$ method. Percentage error is defined as (estimate or bound $-\mathrm{CN}$ result)/ $\mathrm{CN}$ result $\times 100 \%$. Number of images summed: $n=-2$ to $n=2$. Input parameters are: $k=1, \mu=0.1, \sigma=0.2, r=0, S_{u}=110, S_{L}=90, S_{m}=100$ and $S=105$.

\begin{tabular}{|c|c|c|c|c|c|c|}
\hline \multirow{2}{*}{ Duration } & \multirow{2}{*}{$\begin{array}{l}\text { Crank-Nicolson } \\
\qquad \begin{array}{c}\Delta \mathrm{t}=0.0001 \\
\Delta \mathrm{x}=0.0001\end{array}\end{array}$} & \multicolumn{3}{|c|}{$\begin{array}{c}\text { Single-Stage Approximation } \\
\text { (\%error) }\end{array}$} & \multicolumn{2}{|c|}{$\begin{array}{c}\text { Multistage Approximation } \\
\text { (\%error) }\end{array}$} \\
\hline & & Optimal Track & Upper Bound & Lower Bound & Two Stage & Four Stage \\
\hline $\mathrm{T}=0.25$ & 0.748113 & $\begin{array}{l}0.747798 \\
(-0.04 \%)\end{array}$ & $\begin{array}{c}0.750073 \\
(0.26 \%)\end{array}$ & $\begin{array}{l}0.740829 \\
(-0.97 \%)\end{array}$ & $\begin{array}{c}0.748612 \\
(0.07 \%)\end{array}$ & $\begin{array}{c}0.748243 \\
(0.02 \%)\end{array}$ \\
\hline $\mathrm{T}=0.5$ & 0.918689 & $\begin{array}{l}9.919373 \\
(0.07 \%)\end{array}$ & $\begin{array}{c}0.925412 \\
(0.73 \%)\end{array}$ & $\begin{array}{l}0.900014 \\
(-2.03 \%)\end{array}$ & $\begin{array}{l}0.920311 \\
(0.18 \%)\end{array}$ & $\begin{array}{c}0.919099 \\
(0.04 \%)\end{array}$ \\
\hline $\mathrm{T}=0.75$ & 0.973659 & $\begin{array}{c}0.975253 \\
(0.16 \%)\end{array}$ & $\begin{array}{c}0.980940 \\
(0.75 \%)\end{array}$ & $\begin{array}{l}0.952110 \\
(-2.21 \%)\end{array}$ & $\begin{array}{c}0.975566 \\
(0.20 \%)\end{array}$ & $\begin{array}{c}0.974137 \\
(0.05 \%)\end{array}$ \\
\hline$T=1$ & 0.991466 & $\begin{array}{l}0.992988 \\
(0.15 \%)\end{array}$ & $\begin{array}{l}0.996216 \\
(0.48 \%)\end{array}$ & $\begin{array}{l}0.972380 \\
(-1.93 \%)\end{array}$ & $\begin{array}{l}0.992920 \\
(0.15 \%)\end{array}$ & $\begin{array}{l}0.991844 \\
(0.04 \%)\end{array}$ \\
\hline
\end{tabular}

